\title{
SAR Images Target Recognition Based on Wavelet and KSVD
}

\author{
Lei Liu, Xiang-Wei Meng, Zhao-Gen Zhong, Ke-Yuan Yu \\ Department of Electronic and Information Engineering, Naval Aeronautical and Astronautical University, Yantai ,China \\ E-mail: ythyliulei@sina.com, zhongzg@163.com, mengxw@163.com, yuky@163.com
}

\begin{abstract}
Ship targets recognition algorithm based on wavelet domain Kernel Singular Value Decomposition(KSVD) feature extraction was proposed to deal with the problem of ship targets recognition in SAR images. Low-frequency sub-band image is obtained by two-dimension discrete wavelet decomposition of a SAR image. Then it acquires the nonlinear algebraic feature of SAR images by performing KSVD. Support vector machine is used to perform target recognition. The method is applied for recognizing three-class ship targets and the average recognition arrives at $93.89 \%$. It is concluded that the method proposed in this paper is an effective method for SAR images feature extraction and target recognition.
\end{abstract}

Keywords-synthetic aperture radar(SAR); feature extraction; target recognition; kernel singular value decomposition(KSVD); wavelet

\section{INTRODUCTION}

Synthetic Aperture Radar is a kind of active microwave remote sensors. SAR image target recognition is an important part of SAR image interpretation and analysis and it has been widely applied in the field of military reconnaissance and civil observation. Feature extraction is the key link in SAR image target recognition ${ }^{[1][2]}$. How to extract the features quickly and efficiently in the image directly affects the result of target recognition. There are many kinds of feature extraction method applied to SAR image target recognition. Singular Value Decomposition is an effective tool to solve the least squares problem which is a typical linear algorithm. Images which are obtained by singular value decomposition characteristics have high stability and therefore it has been widely applied in the field of target recognition. While SVD transform is a linear algorithm and it can not extract the nonlinear structural features in the image, which affects the identification accuracy $^{[3]}$.

This paper proposes a feature extraction method based on wavelet domain Kernel Singular Value Decomposition. Extract the low-frequency sub-band image by two-dimension discrete wavelet decomposition and then acquire the nonlinear algebraic feature of SAR images by performing KSVD. Finally complete the target recognition with the SVM classifier. Using three kinds of ship target image from RADARSAT-2 to verify and the results show that the method in this paper an effective method.

\section{KERNEL SINGULAR VALUE DECOMPOSITION}

A. Singular Value Decomposition

Singular value decomposition theorem ${ }^{[4-6]}$ : set $\boldsymbol{a} \in \boldsymbol{R}^{m \times n}(m>n), \quad \operatorname{rank}(\boldsymbol{a})=r$. There are orthogonal matrix $\boldsymbol{U}=\left[\boldsymbol{u}_{1}, \boldsymbol{u}_{2}, \cdots, \boldsymbol{u}_{m}\right] \in \boldsymbol{R}^{m \times m}, \boldsymbol{V}=\left[\boldsymbol{v}_{1}, \boldsymbol{v}_{2}, \cdots, \boldsymbol{v}_{n}\right] \in \boldsymbol{R}^{n \times n}$ and diagonal matrix $\Sigma=\operatorname{diag}\left[\sigma_{1}, \sigma_{2}, \cdots \sigma_{r}, 0, \cdots, 0\right] \in \boldsymbol{R}^{m \times n}$, $\sigma_{1}>\sigma_{2}>\cdots>\sigma_{r} \geq 0$ to make

$$
\boldsymbol{a}^{r}=\boldsymbol{U}^{r} \Sigma^{r}\left(\boldsymbol{V}^{r}\right)^{T}
$$

$\boldsymbol{U}^{r}, \boldsymbol{V}^{r}$ are the first $r$ column of $\boldsymbol{U}, \boldsymbol{V}$ respectively.

Using the space composed by $\boldsymbol{U}^{r}$ to express the target in the image. $\boldsymbol{U}^{r}$ is a orthogonal basis of $r$ dimension subspace .It is minimized

$$
\left\|a-a^{r}\right\|_{F}
$$

In the type, $\boldsymbol{a}^{r}=\boldsymbol{U}^{r}\left(\boldsymbol{U}^{r}\right)^{T} \boldsymbol{a}$ and it is the approximation of $r ;\|\cdot\|_{F}$ is the Frobenius norm.

The main component of $\boldsymbol{a}$ is obtained after centralized treatment and singular value decomposition of the vector in $\boldsymbol{a}$.

\section{B. Kernel Singular Value Decomposition}

Singular value decomposition belongs to linear algorithm, so it can't extract nonlinear structural features in the data. The nonlinear characteristic exists widely in the practical problems. Therefore, we introduce the skill of kernel ideas. Data will be mapped to high-dimensional space with a nonlinear transformation $\varphi: \boldsymbol{R}^{m} \rightarrow \boldsymbol{F}$ and carries on the singular value decomposition in high dimensional space. Select the appropriate kernel function $k(\bullet, \bullet)$ to realize the nonlinear mapping $\varphi^{[7]}$.

$\boldsymbol{a}$ can be transformed to $\boldsymbol{A}=\left[\varphi\left(x_{1}\right) \cdots \varphi\left(x_{n}\right)\right]$ through $\varphi$. $\boldsymbol{M}=\boldsymbol{A}^{\mathrm{T}} \boldsymbol{A}$ and its eigenvalue decomposition is $\boldsymbol{M}=\boldsymbol{Q} \Delta \boldsymbol{Q}^{\mathrm{T}}$. Singular value decomposition of $\boldsymbol{A}$ is

$$
\boldsymbol{A}^{r}=\left[\boldsymbol{A} \boldsymbol{Q}^{r}\left(\Delta^{r}\right)^{-\frac{1}{2}}\right]\left[\left(\Delta^{r}\right)^{\frac{1}{2}}\right]\left[\left(\boldsymbol{Q}^{r}\right)^{T}\right] \equiv \boldsymbol{U}^{r} \Sigma^{r}\left(\boldsymbol{V}^{r}\right)^{T}
$$

$\boldsymbol{Q}^{r}$ is the first $r$ column of $\boldsymbol{Q} ; \Delta^{r}$ is the cross phalanx of the first $r$ row and $r$ column of $\Delta$. This is the basic 
thought of KSVD method ${ }^{[8]}$

The common kernel function ${ }^{[9]}$ is as follows:

(1) Polynomial kernel function

$$
K\left(x, x^{\prime}\right)=\left\langle x, x^{\prime}\right\rangle^{d}
$$

$x, x^{\prime} \in \mathrm{R}^{m} ; d$ is an integer.

(2) Gaussian radial basis kernel function

$$
K\left(x, x^{\prime}\right)=\exp \left(-\frac{\left\|x-x^{\prime}\right\|^{2}}{2 \delta^{2}}\right)
$$

$\delta$ is the width of the kernel.

(3) Index radial basis kernel function

$$
K\left(x, x^{\prime}\right)=\exp \left(-\frac{\left\|x-x^{\prime}\right\|}{2 \delta^{2}}\right)
$$

(4) Sigmoid kernel function

$$
K\left(x, x^{\prime}\right)=\tanh \left(p\left\langle x, x^{\prime}\right\rangle+c\right)
$$

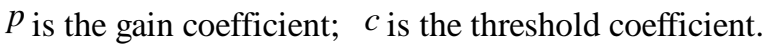

\section{FEATURE EXTRACTION METHOD BASED ON WAVELET AND KSVD}

\section{A. Two-dimensional Discrete Wavelet Transform}

The target images are decomposed on different scales and extract the low-frequency sub-band images[10]. Its expression is:

$$
f(n)=\sum_{k \in Z^{2}} a_{j_{0}, k} \phi_{j_{0}, k}^{L L}(n)+\sum_{b \in B} \sum_{j \geq j_{0}} \sum_{k \in Z^{2}} d_{j, k}^{b} \varphi_{j, k}^{b}(n)
$$

$\phi_{j_{0}, k}^{L L}(n)$ is two-dimensional scale function; $\varphi_{j, k}^{b}(n)$ is two-dimensional wavelet function; $a_{j_{0}, k}$ is the scale factor; $d_{j, k}^{b}$ is the wavelet coefficients. $b \in B=\{\mathrm{LH}, \mathrm{HL}, \mathrm{HH}\} ; j$ is scale factor; $j_{0}$ is fixed scale; $k$ is two-dimensional displacement factor. LL is the low-frequency component, $\mathrm{LH}, \mathrm{HL}$ and $\mathrm{HH}$ are horizontal component, vertical component and the diagonal component respectively.

In this paper, the pixel of the low-frequency sub-band image is only $1 / 64$ of the original target image, but it contains most of the original image information. The high-frequency sub image contains the speckle noise in images. The wavelet decomposition realizes the image compression and removes the noise of the image.

\section{B. Kernel Singular Value Decomposition}

Low-frequency sub-band images are obtained by wavelet transform. Then the data is normalized and all the properties are put into $[1,1]$ by linear transformation. The normalized data is put into high dimension space by kernel function and obtain the kernel matrix. This paper uses the $\sigma=100$ Gaussian radial basis kernel function to realize the nonlinear mapping. The nonlinear algebraic features are as the image classification features.

\section{Ship Targets Recognition Based On Support Vector Machine}

In this paper, container ship, oil tanker and cargo ship from RADARSAT 2 image are used for classification. Set

the training sample $\left(e_{i}, Z_{i}\right), i=1, \cdots, m, e_{i} \in R^{r}$ is the characteristic vector after wavelet decomposition and kernel singular value decomposition. $z_{i} \in\{1,2,3\}$ is a class label. $m$ is the total number of three kinds of ship target. $r$ is dimensions of the feature vector after the singular value decomposition. Support vector machine can only be used for classification between two samples. This paper need to classify three kinds of ship targets, so it selects the voting strategy of "one to one". Using ballot form and the most votes in the class is the test sample's category. Recognition process is shown in figure 1 .

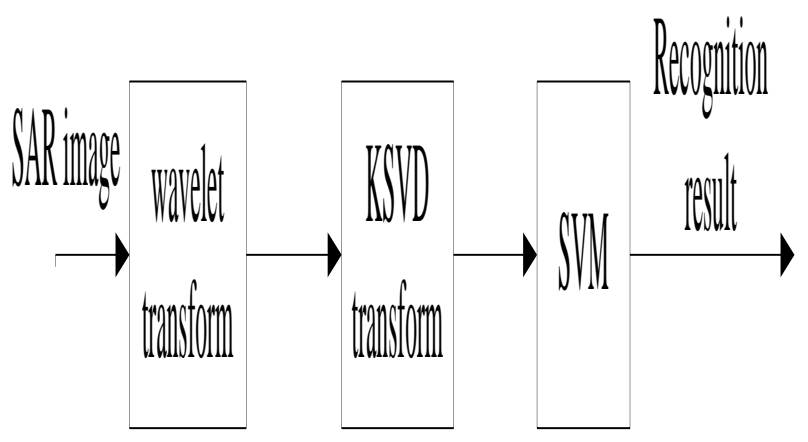

Figure 1. Flowchart of recognition.

\section{EXPERIMENT AND ANALYSIS}

Use the ship target images from RADARSAT-2 for experiment. Choose vessels including 130 container ships, 130 oil tankers and 130 cargo ships. Figure 2 shows the optical image and the SAR image of the ships. All SAR image target section size is $128 \times 128$. The first 70 images of each type of the ships are as the training images and the others are as test samples. 

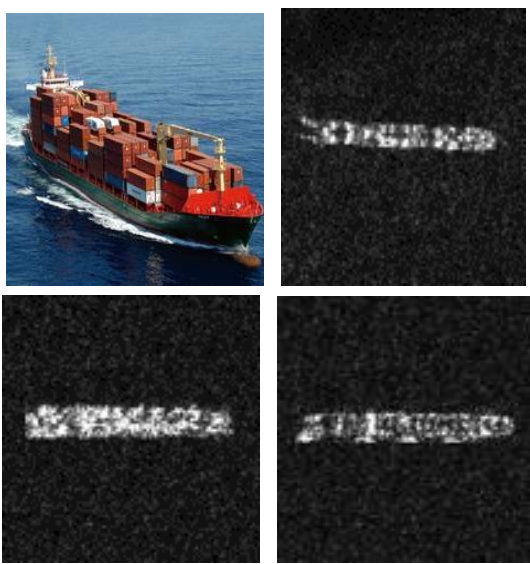

Container ship

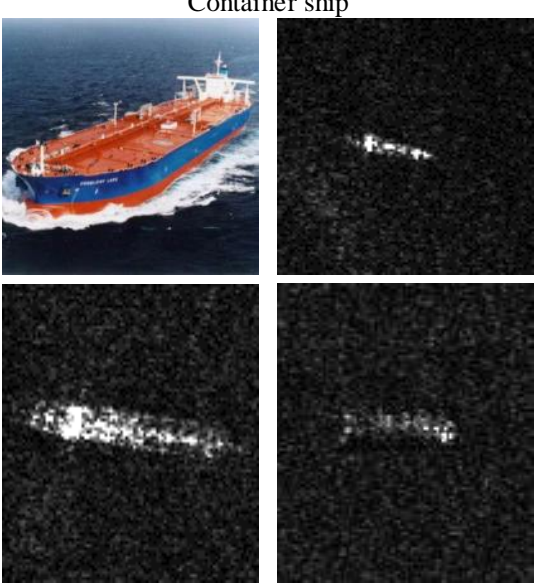

Oil tanker

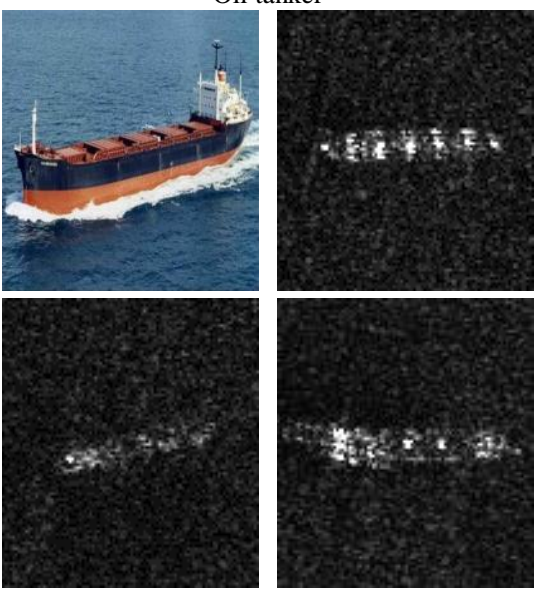

Cargo ship

Figure 2. Ship sample of SAR image.

The first cargo ship is been as an example. Put it into two-dimensional discrete wavelet decomposition using $\mathrm{db} 2$ wavelet basis. Two-dimensional wavelet decomposition diagram is shown in figure 3. (a), (b), (c) are one layer, two layer and three layer wavelet decomposition respectively. The image after three layer wavelet transformation of is numbered as shown in (d). Table 1 show the energy percentage of the original image that each sub image contained.

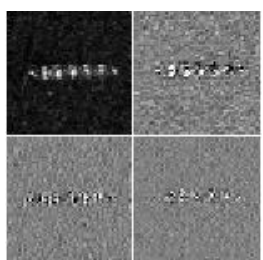

(a)

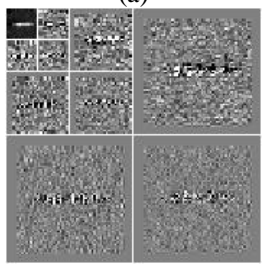

(c)

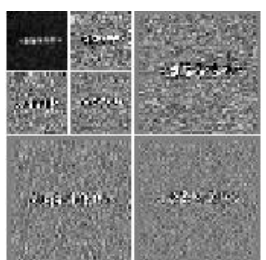

(b)

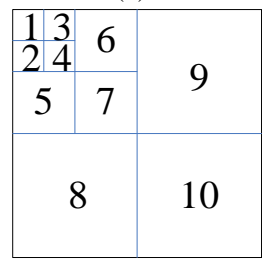

(d)
Figure 3. Two-dimension wavelet decomposition.

TABLE I. ENERGY DISTRIBUTION OF THREE PHASES WAVELET DECOMPOSITION

\begin{tabular}{cccccc}
\hline & pixel & energy(\%) & & pixel & energy(\%) \\
\hline $\mathbf{1}$ & $16 \times 16$ & 74.92 & $\mathbf{2}$ & $16 \times 16$ & 3.51 \\
$\mathbf{3}$ & $16 \times 16$ & 5.90 & $\mathbf{4}$ & $16 \times 16$ & 1.99 \\
$\mathbf{5}$ & $32 \times 32$ & 3.41 & $\mathbf{6}$ & $32 \times 32$ & 3.13 \\
$\mathbf{7}$ & $32 \times 32$ & 1.43 & $\mathbf{8}$ & $64 \times 64$ & 3.56 \\
$\mathbf{9}$ & $64 \times 64$ & 1.28 & $\mathbf{1 0}$ & $64 \times 64$ & 0.88 \\
\hline
\end{tabular}

It can be seen from table 1 that the image dimension is from $128 \times 128$ to $16 \times 16$ after three layer wavelet decomposition, but it contains $74.92 \%$ information of the original image. The amount of data is reduced.

The low-frequency sub-band images are extracted after three layer wavelet decomposition and each image is connected to 256 dimension column vectors by column. Extract the feature vector of the sub-band images with PCA, KPCA, SVD and KSVD method. Tthe target recognition rate of these methods were compared shown in table 2 .

TABLE II. COMPARISON OF THE RECOGNITION RATE

\begin{tabular}{ccccc}
\hline & $\begin{array}{c}\text { cargo } \\
\text { ship }\end{array}$ & $\begin{array}{c}\text { oil } \\
\text { tanker }\end{array}$ & $\begin{array}{c}\text { container } \\
\text { ship }\end{array}$ & $\begin{array}{c}\text { recognition } \\
\text { rate }\end{array}$ \\
\hline $\begin{array}{c}\text { Wavelet and } \\
\text { PCA }\end{array}$ & 86.67 & 86.67 & 90.00 & $87.78 \%$ \\
$\begin{array}{c}\text { Wavelet and } \\
\text { KPCA }\end{array}$ & 90.00 & 88.33 & 93.33 & $90.55 \%$ \\
$\begin{array}{c}\text { Wavelet and } \\
\text { SVD }\end{array}$ & 91.67 & 90.00 & 95.00 & $92.22 \%$ \\
$\begin{array}{c}\text { Wavelet and } \\
\text { KSVD }\end{array}$ & 93.33 & 91.67 & 96.67 & $93.89 \%$ \\
\hline
\end{tabular}

It can be seen from table 2 that recognition result of this paper's method is better than the wavelet transform and PCA, SVD, and KPCA. Ship target recognition rate is $93.89 \%$ and ship target recognition rate is improved effectively.

\section{CONCLUSIONS}

This paper proposes a ship targets recognition algorithm based on wavelet domain kernel singular value decomposition. First using two-dimensional discrete 
wavelet transforms to compress the image while remove the high frequency noise in the image and obtain the low-frequency sub-band image. Then it acquires the nonlinear algebraic feature of SAR images by performing KSVD. Support vector machine is used to perform target recognition. The experimental results show that the algorithm can get high recognition rate and is an effective method for SAR image feature extraction and target recognition.

\section{REFERENCES}

[1] LI Y, GONG H L, LIANG J X et al. SAR image target feature extraction based on KSVD and PCA[J]. Journal of Jilin University (Engineering and Technology Edition), 2010,40(5):1136-1139.

[2] HUAN R H, YANG R L. Synthetic Aperture Radar Images Target Recognition Based on Wavelet Domain NMF Feature Extraction[J]. Journal of Electronics \& Information Technology, 2009,31(3):588-591.

[3] LI H L, SU F L. Space Target Recognition Algorithm Based on Wavelet Transform and Singlular Value[J]. Electronics Optics \& Control,2009,16(3):29-32.
[4] Akritas A G, Malaschonok G I. Applications of singular value decomposition (SVD)[J]. Mathematics and Computers in Simulation,2004,67(1):15-31.

[5] ZHAO X Z, YE B Y. The Similarity of Signal Processing Effect between SVD and Wavelet Transform and Its Mechanism Analysis[J]. Acta Electronica Sinica, 2008,36(8):1582-1589.

[6] ZHAO X Z, YE B Y. Multi-resolution SVD Packet Theory and Its Application to Signal Processing[J].Acta Electronica Sinica,2012,40(10):2039-2046.

[7] CHIN T J, SCHINDLER K, SUTER D. Incremental kernelSVD for face recognition with image sets[C]. Proceedings of the 7th International Conference on Automatic Face and Gesture Recognition (FGR), So ut hampton, United Kingdom, 2006.

[8] CRISTIANINI N, SHAWE $\mathrm{T}$ J. Kernel Methods for Pattern Analysis[M],England: Cambridge University Press,2004.

[9] SANDIRASEGARAM N and ENGLISTH R. Comparative analysis of feature extraction (2D FFT and wavelet) and classification(Lp metric distances, MLP NN, and HNeT) algorithms for SAR imagery. Proc. SPIE, 2005, 5808:314-325.

[10] CHEN W F. The Wavelet analysis and its application in image processing[M]. Beijing: SciencePress,2002. 